Reprod. Nutr. Develop., 1988, 28 (3 B), 703-713

\title{
Les stratégies moléculaires impliquées dans le contrôle de l'expression des gènes au cours de la différenciation des cellules musculaires
}

\author{
M. Y. FISZMAN
}

Unité de Biochimie, Département de Biologie moléculaire, Institut Pasteur, 25, rue du Dr Roux, 75724 Paris Cedex 15.

Summary. Molecular strategies involved in the control of gene expression during muscle cell differentiation.

The initial step in muscle formation is the fusion of undifferentiated myoblasts into multinucleated myotubes which synthesize the specific proteins of the muscle. During this transition a whole series of genes are turned on. A simple explanation for this activation process is that each gene contains a structure which is common to all genes of the family and which is recognized by (a) specific factor(s). Experimental evidence supporting this model is described.

Appearance of a new protein may not involve gene activation but be the result of a new mode of splicing as in the case of tropomyosins.

Examples have been chosen to illustrate the various strategies used to control gene expression and provide the necessary diversity required during the process of muscle maturation. They include controlled expression of linked genes, coexpression of genes from unrelated phenotypes or alternative splicing of unique genes.

Le système musculaire représente un système de choix pour l'étude d'un processus de différenciation terminale. Tout d'abord, il faut bien reconnaître que la formation des fibres multinuclées à la suite de la fusion des myoblastes entre eux représente un des exemples les plus spectaculaires de différenciation morphologique. Ensuite, le fait qu'il soit possible d'obtenir cette étape de différenciation en culture, permet d'envisager d'aborder au plan moléculaire les mécanismes qui contrôlent l'ensemble de ce processus. C'est ce système de cellules en culture qui va nous servir de base pour l'analyse des stratégies moléculaires développées au cours de la myogenèse.

\section{Différenciation des myoblastes en culture.}

\section{1) Rappel.}

A partir de muscle embryonnaire on peut préparer des cultures primaires de myoblastes. Ces myoblastes, pourvu qu'on leur fournisse un milieu nutritif 
adéquat, vont se multiplier pendant un nombre de générations qui dépend à la fois de l'espèce utilisée et de la densité d'ensemencement des cellules. Cette phase de multiplication va être suivie d'une phase durant laquelle les myoblastes sortent du cycle cellulaire, s'organisent les uns par rapport aux autres pour finalement fusionner entre eux pour former les structures syncitiales que sont les myotubes. Sur le plan biochimique, les myoblastes ne présentent pas de phénotype caractéristique alors que les myotubes sont caractérisés par le fait qu'ils synthétisent les protéines spécifiques du muscle.

Quand on analyse la cinétique d'apparition des protéines spécifiques du muscle au cours de la formation des myotubes on constate d'une part qu'elles s'accumulent à la suite de la fusion et d'autre part que leur synthèse semble être coordonnée. En ce qui concerne le premier point, on sait maintenant qu'il n'existe aucune relation entre le phénomène de fusion et l'apparition des protéines du muscle et qu'il s'agit de deux caractères indépendants du phénotype différencié.

\section{2) Recrutement coordonné des gènes spécifiques du phénotype musculaire.}

L'utilisation de sondes moléculaires clonées a permis de montrer que l'apparition des protéines spécifiques du muscle correspond à une accumulation des ARN messagers correspondant. De plus, l'utilisation de techniques comme celle mesurant la sensibilité des gènes à la DNase 1 ou celles qui, de manière plus directe, visualisent les gènes en cours de transcription, permet de conclure que le contrôle de l'apparition des protéines spécifiques du muscle se fait au niveau de la transcription. En d'autres termes, les gènes codant pour ces protéines seraient inactifs chez les myoblastes et commenceraient à être transcrits de manière coordonnée quand les myoblastes sont induits à se différencier.

Comment explique-t-on ce phénomène de commutation multiple qui se produit lors de la transition myoblaste-myotube? La figure 1 présente une des hypothèses que l'on peut proposer. Comme on le voit, ce modèle repose sur l'existence de deux éléments essentiels : 1) un (ou des) facteur(s) agissant en trans et 2) un motif particulier commun à tous les gènes contribuant au phénotype musculaire.

Existe-t-il des preuves expérimentales de ce modèle?

A) Activation artificielle des gènes musculaires. - Une des preuves les plus convaincantes de l'existence de ce(s) facteur(s) agissant en trans a été apportée par le groupe du professeur $\mathrm{H}$. Blau. Ces auteurs ont réalisé une fusion artificielle entre des cellules non musculaires humaines et des cellules musculaires différenciées de souris. Quand on analyse les hétérocaryons formés, on constate qu'il y a accumulation de protéines spécifiques de muscle humain. Autrement dit quand des noyaux d'une cellule non musculaire sont introduits dans un cyoplasme où s'exprime le phénotype musculaire, il y a activation des gènes correspondant au phénotype musculaire grâce à des facteurs présents dans ce cytoplasme (Blau et al., 1983, 1985 ; Chiu et Blau, 1984 ; Hardeman et al., 1986).

Une autre approche expérimentale a été de montrer qu'il est possible d'induire l'expression de phénotypes nouveaux chez des cellules fibroblastiques 


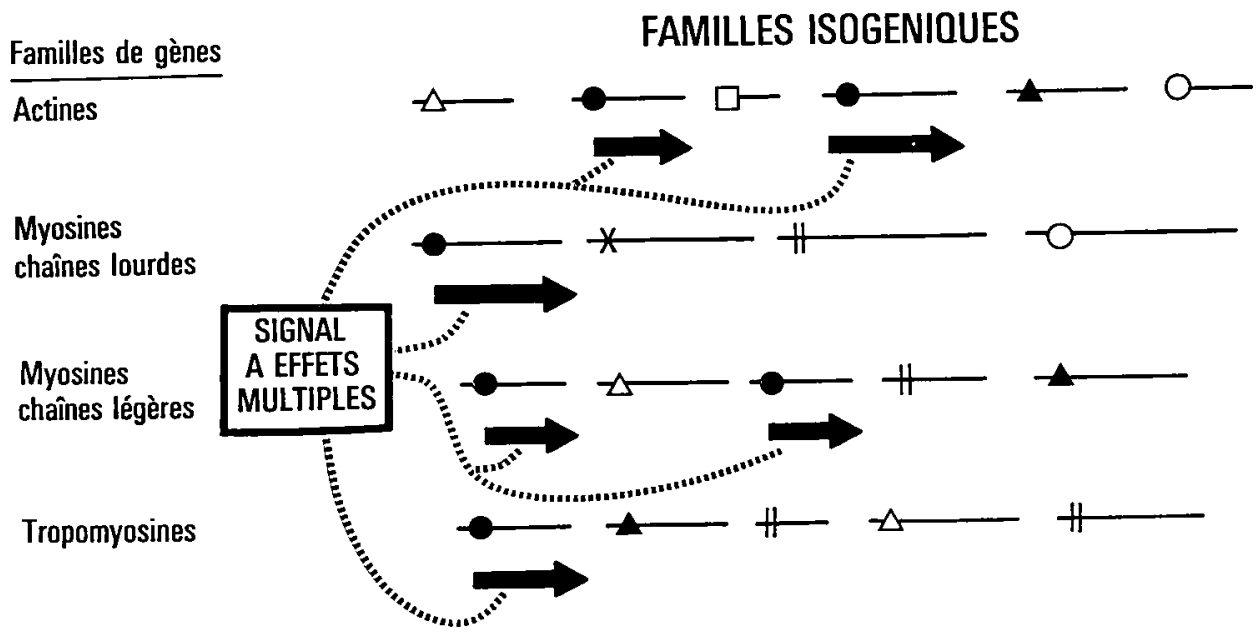

FIG. 1. - Modèle de régulation coordonnée de l'expression d'une famille de gènes. Les symboles identiques signalent l'appartenance des gènes à un même phénotype.

de souris, les cellules C3H 10T1/2. Pour cela, il suffit de traiter ces cellules par de la 5 Azacytidine. Ce produit prend la place de la cytidine et de ce fait empêche les processus de méthylation d'avoir lieu, entraînant une hypométhylation de l'ADN. Dans ces conditions, on constate qu'à partir des cellules fibroblastiques, on peut isoler des clones de cellules exprimant un phénotype musculaire ou chondrocytaire ou adipocytaire (Taylor et Jones, 1979, 1982 ; Konieczny et Emerson, 1984). En d'autres termes, on peut, du fait de l'hypométhylation de l'ADN, transformer un fibroblaste en myoblaste, chondroblaste ou adipocyte. Ce qui est particulièrement intéressant dans cette expérience, c'est que la fréquence avec laquelle apparaissent ces nouveaux phénotypes est compatible avec l'hypothèse qu'il suffit de modifier un seul gène pour que le nouveau phénotype s'exprime. Il est alors tentant d'imaginer que le gène ainsi modifié est celui qui code pour ce(s) facteur(s) d'activation dont nous venons de parler. Mais alors, si cette hypothèse est correcte, rien n'empêche d'imaginer qu'on puisse introduire artificiellement ce gène modifié dans une cellule quelconque et par là, la transformer en cellule musculaire. Différents auteurs ont ainsi transfecté de l'ADN génomique de cellule musculaire dans des cellules $10 \mathrm{~T} 1 / 2$ et sont parvenus à isoler des clones de cellules myogéniques (Lassar et al., 1986). Le clonage des séquences ainsi transfectées est en cours et on peut penser que dans un bref avenir on saura exactement ce pour quoi elles codent.

L'ensemble de ces résultats étaie de manière assez convaincainte l'idée que des facteurs agissant en trans sont impliqués dans le recrutement de l'ensemble des gènes qui codent pour les caractères spécifiques du phénotype musculaire. La question se pose alors de savoir avec quelle séquence des gènes ces facteurs interviennent. Quoiqu'on soit encore loin de pouvoir répondre avec précision à cette question, on commence cependant à posséder une série de résultats expérimentaux qui permettent de préciser la localisation de ces séquences. 
B) Séquences régulatrices en $5^{\prime}$ des gènes. - Deux protocoles expérimentaux sont actuellement utilisés pour localiser les séquences régulatrices étant bien entendu que la simple comparaison des séquences nucléotidiques a jusqu'à présent été passablement décevante.

- Expression transitoire : Une première méthode a consisté à isoler un fragment du gène situé en $5^{\prime}$ de l'origine de transcription, à le rattacher au gène bactérien codant pour le chloramphénicol acétyltransférase (CAT) et à transfecter la construction soit dans des cellules non musculaires, soit dans des cellules musculaires différenciées ou non. 24 à $48 \mathrm{~h}$ après la transfection on mesure la production de l'enzyme CAT, lequel est naturellement absent des cellules animales. Si la région $5^{\prime}$ utilisée contient un promoteur actif, on détectera une activité CAT, sinon l'essai sera négatif. Ce système permet non seulement d'avoir une réponse qualitative mais également une réponse partiellement quantitative.

Ce type d'approche a été utilisée avec succès dans de nombreux cas et a permis de délimiter une région minimum contenant des séquences régulatrices reconnues spécifiquement dans des cellules musculaires (Melloul et al., 1984 ; Nudel et al., 1985 ; Bergsma et al., 1986a ; Minty et al., 1986a ; Siddiqui et al., 1986). D'une manière générale, on constate que ces séquences sont localisées dans une région relativement limitée recouvrant environ 500 bp en $5^{\prime}$ du site d'initiation de la transcription. Ainsi Schwartz et ses collaborateurs (Bergsma et al., 1986b) ont montré qu'avec le gène de l' $\alpha$ actine squelettique 200 nucléotides en $5^{\prime}$ sont suffisants pour obtenir une activité CAT maximale. Certains auteurs ont également mis en évidence des séquences multiples. Par exemple, Minty et ses collaborateurs ont observé avec l' $\alpha$ actine cardiaque qu'un fragment de $450 \mathrm{nu}$ cléotides permet d'obtenir une activité CAT maximale. Si ce fragment est délété de 50 nucléotides, l'activité est réduite de $40 \%(-443$ à - 395) et ce n'est que si on délète en aval de la position - 180 que l'activité est réduite de $98 \%$ (- 177 à - 47) (Minty et Kedes, 1986).

Il est important d'observer que la signification et le rôle exact de ces séquences n'est pas encore complètement compris. En effet, si les résultats obtenus sont clairs en ce qui concerne les comparaisons entre cellules non musculaires (pas d'activité CAT) et cellules musculaires (activité CAT), des résultats conflictuels ont été obtenus pour les comparaisons entre cellules musculaires différenciées et non différenciées. Ainsi Minty et al. ont-ils montré que la même construction qui donne une activité CAT avec les cellules C2 différenciées ou non, ne donne d'activité CAT qu'avec les cellules L8 différenciées mais pas avec les cellules L8 non différenciées (Minty et al., 1986b). Ce résultat montre donc que selon la lignée cellulaire utilisée, les facteurs agissant en trans sont ou non présents avant la différenciation. Cependant, il faut bien noter que lorsqu'on obtient une activité CAT dans des cellules à l'état myoblaste, au même moment le gène endogène n'est pas transcript. Ce type de considération a fait proposer à Minty et à ses collaborateurs qu'il doit exister deux niveaux de régulation, l'un lié à l'activation du gène qui est responsable de la capacité de ce gène a être transcrit et l'autre qui contrôle le niveau auquel se fait cette transcription. Ainsi chez les myoblastes $\mathrm{C} 2$ le gène endogène n'est pas activé et 
n'est donc pas transcrit alors que la construction transfectée est capable d'être transcrite et comme les facteurs de modulation sont présents, elle est transcrite activement d'où l'activité CAT mesurée. Pour ces auteurs, les expériences d'expression transitoire permettraient donc de mettre en évidence la présence des facteurs de modulation, mais ne permettraient pas d'appréhender le phénomène d'activation proprement dit.

Des résultats en accord avec cette conclusion ont également été obtenus en étudiant l'expression transitoire de gènes entiers transfectés (Seiler-Tuyns et al., 1984). Dans cette expérience trois gènes actine ont été transfectés: $\beta$-cytoplasmique, $\alpha$-cardiaque et $\alpha$-squelettique. En ce qui concerne le gène $\beta$, il est non seulement correctement transcrit dans les cellules non musculaires mais en plus il subit une régulation négative dans les cellules musculaires différenciées exactement comme le gène endogène. Au contraire pour les deux gènes $\alpha$-actine, s'ils sont essentiellement transcrits dans les cellules musculaires, ils ne subissent aucune régulation en fonction de l'état de différenciation des cellules.

- Transfection stable: Une deuxième méthode a consisté à transfecter soit le gène entier soit la région promotrice couplée à un gène marqueur mais cette fois on va sélectionner des transfectants stables. Les expériences ont été réalisées soit avec des cellules myogéniques (Nudel et al., 1985), soit avec les cellules 10T1/2 précédemment décrites (Konieczny et Emerson, 1985) soit enfin avec des zygotes ce qui permet d'obtenir des animaux transgéniques (Shani, 1985). Dans tous les cas on constate que les régions définies dans les expériences précédentes suffisent pour permettre une régulation parfaite de l'expression des gènes $y$ compris lors de la transition myoblaste-myotube. Ceci pourrait alors suggérer que le phénomène d'activation impliquerait plutôt une conformation que des séquences proprement dites. A ce sujet il est intéressant de mentionner une expérience récente du groupe de Yaffe (Yisraeli et al., 1986). Ces auteurs ont transfecté des constructions $5^{\prime} \alpha$-actine squelettique/CAT arprès les avoir artificiellement méthylées et ils constatent que ces constructions sont spécifiquement déméthylées dans les cellules myogéniques mais pas dans des cellules non musculaires. Ce résultat ferait penser que les processus de déméthylation jouent un rôle important dans le phénomène d'activation.

\section{3) Utilisation de gènes transcrits de manière constitutive.}

Jusqu'à présent nous n'avons envisagé que le cas de gènes activés lors de la transition myoblaste-myotube, or des résultats récents indiquent que des gènes codant pour des protéines musculaires peuvent également coder pour des protéines non musculaires, c'est en particulier le cas des gènes codant pour des sous-unités $\alpha$ et $\beta$ de la tropomyosine de muscle du squelette (Ruiz-Opazo et Nadal-Ginard, 1987 ; Helfman et al., 1986 ; Fiszman et al., 1986 ; Libri et al., 1988). Que se passe-t-il pour ces gènes au cours de la différenciation?

A titre d'exemple, nous envisagerons le cas du gène de la $\beta$-tropomyosine. Ce gène code pour la $\beta$-tropomyosine de muscle lisse et pour celle de muscle du squelette grâce à un mécanisme d'épissage alternatif (fig. 2). Ainsi qu'on peut le 

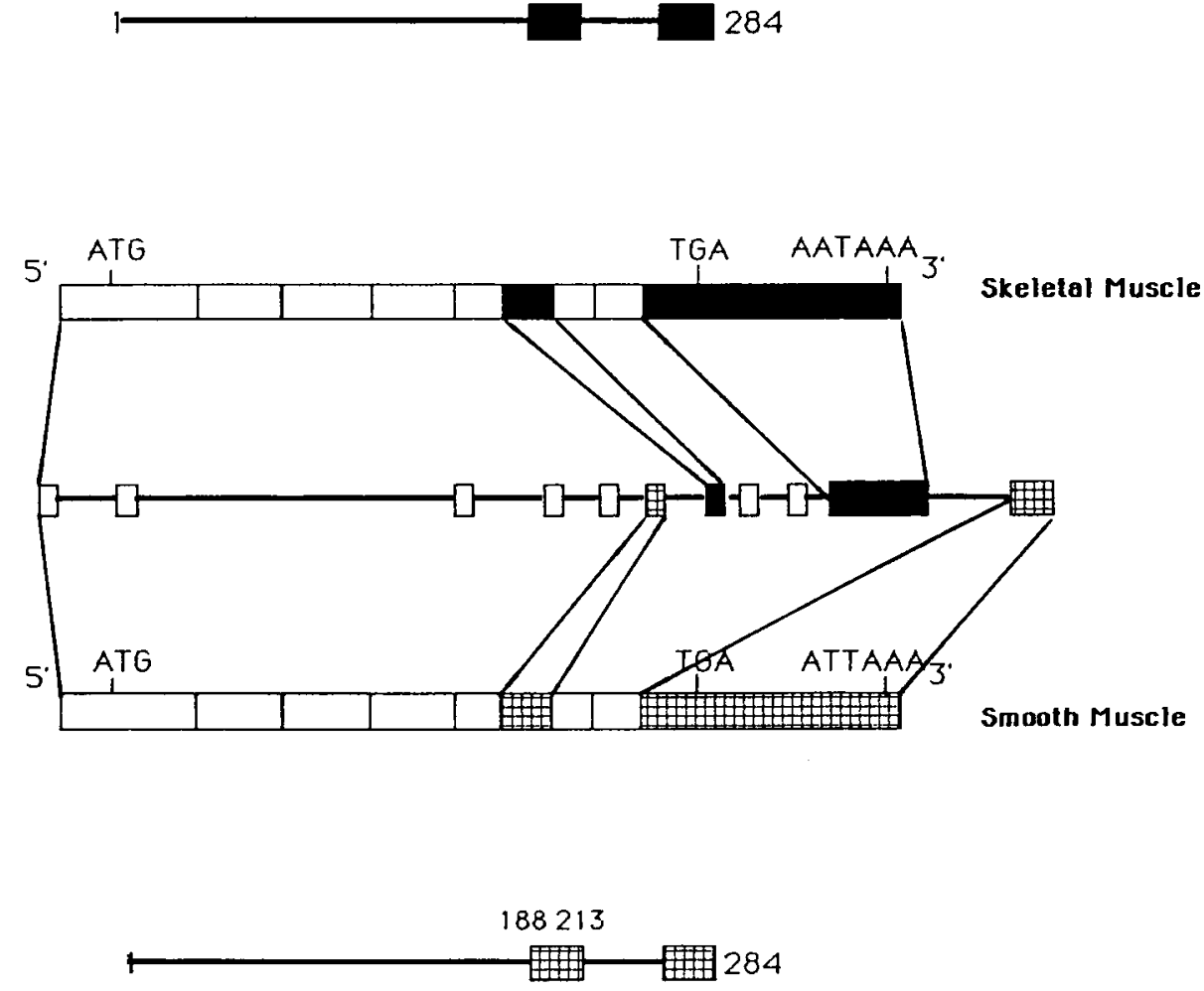

FIG. 2. - Représentation schématique de lorganisation du gène de la $\beta$-tropomyosine et des deux modes d'épissage.

voir sur la figure 3, les myoblastes accumulent un ARN messager de $1.3 \mathrm{~kb}$ qui correspond à la $\beta$-tropomyosine de muscle lisse. Quand les myoblastes se différencient, on voit s'accumuler un ARN messager de $1.6 \mathrm{~kb}$ qui correspond à la $\beta$-tropomyosine musculaire. On peut également noter que le messager de $1.3 \mathrm{~kb}$ tend à disparaître ce qui traduirait un phénomène de régulation négative. Quoiqu'il en soit, on voit donc que ce gène s'exprime déjà au stade myoblaste et qu'au cours de la transition il y a non pas activation du gène mais remplacement d'un mode d'épissage par un autre, ce qui permet l'accumulation de la forme musculaire. Il faut également envisager un mécanisme de contrôle quantitatif puisque I'ARN de $1.6 \mathrm{~kb}$, dans les myotubes, est nettement plus abondant que celui de $1.3 \mathrm{~kb}$ dans les myoblastes. Ceci peut soit impliquer des différences de stabilité des deux ARN messagers soit une augmentation de la transcription avec intervention des séquences de modulation dont nous avons déjà parlé et qui seraient localisées dans la région du promoteur. Les expériences sont en cours pour résoudre cette alternative. Ce que nous venons de dire pour le gène $\beta$ est également vrai pour le gène de l' $\alpha$-tropomyosine. 


\section{1}

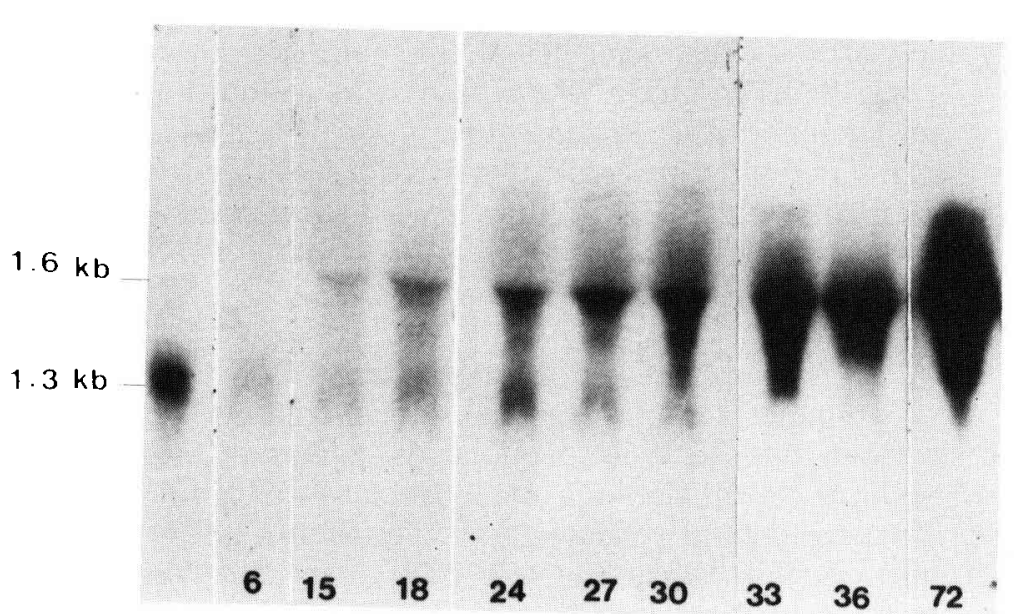

FIG. 3. - Cinétique d'accumulation des ARN messagers codant pour les $\beta$-tropomyosines. 35) Température non permissive pour la différenciation; 41) température permissive pour la différenciation ; 6-15-18-24-27-30-33-36-72) heures d'incubation des cellules en condition de différenciation.

\section{Transitions moléculaires au cours du développement.}

On peut décrire le développement du système musculaire comme une suite de transitions tant sur le plan morphologique, métabolique et moléculaire. La plupart de ces transitions ne peuvent être appréhendées que dans l'organisme entier dans la mesure où sont impliquées des interactions tissulaires ou hormonales. De plus, il faut remarquer que certaines des transitions moléculaires observées dans les muscles entiers ne se produisent pas dans les fibres préexistantes mais sont dues à l'apparition de nouvelles populations cellulaires exprimant des phénotypes distincts. Ces transitions ne seront pas analysées dans la suite de notre article puisqu'elles n'impliquent pas de mécanisme de contrôle particulier. On peut considérer trois catégories de transitions : 1) l'expression séquentielle d'une famille de gènes liés (cas des chaînes lourdes de la myosine), 2) la coexpression de gènes appartenant à des phénotypes tissulaires différents (cas des actines et des chaînes légères de la myosine) et 3) l'épissage alternatif d'un gène unique (cas de LC1/LC3).

\section{1) Expression séquentielle de gènes.}

Nombre de travaux ont montré qu'au cours du développement musculaire il apparaît trois formes isomorphes des chaînes lourdes de la myosine : une forme fotale, une forme périnatale et une forme adulte (pour revue: Bandman, 1985). Cette transition existe chez tous les vertébrés, toutefois, les cinétiques diffèrent d'un animal à l'autre et peut être également d'un type musculaire à l'autre (Crow 
et Stockdale, 1986). Grâce au clonage moléculaire, on sait que chaque forme isomorphe est codée par un gène particulier et qui plus est, on a pu montrer, chez la souris, que les trois gènes sont liés et sont localisés sur le chromosome 11 (Weydert et al., 1985). Chez l'embryon, la transition forme fœtale-forme périnatale est indépendante de l'innervation (Weydert et al., 1987). II est intéressant de noter que cet ensemble de transitions peut être observé en culture sans pour autant qu'on puisse comprendre quel en est le mécanisme de régulation (Cerny et Bandman, 1986 ; Silberstein et al., 1986 ; Weydert et al., 1987).

\section{2) Coexpression de gènes.}

Les deux exemples les plus étudiés concernent l'expression de l'actine cardiaque et squelettique et celui des chaînes légères LC1 de la myosine des oreillettes et du muscle squelettique. Dans les deux cas, on a décrit, aussi bien chez l'embryon qu'en culture, l'accumulation d'une forme dite fœtale (Minty et al., 1982) ou embryonnaire (Whalen et al., 1978) précédant l'accumulation de la forme adulte. Dans les deux cas, l'utilisation de sondes clonées a permis de montrer que ces formes dites embryonnaires appartenaient en fait au phénotype cardiaque et pour LC1, plus particulièrement aux oreillettes (Minty et al., 1982 ; Barton et al., 1985). On voit donc qu'au cours du développement musculaire on peut recruter indistinctement des gènes squelettiques ou cardiaques. II faut d'ailleurs noter que le système peut également fonctionner en sens inverse puisque dans certaines situations pathologiques le cœur peut accumuler de l'actine squelettique (Schwartz et al., 1986), ceci est également vrai chez un mutant de la souris où ce mécanisme sert à compenser un défaut d'expression de l'actine cardiaque (Garner et al., 1986).

\section{3) Epissage différenciel d'un même gène.}

Durant la vie embryonnaire le muscle n'accumule que la forme LC1 et ce n'est qu'à partir de la naissance que la forme LC3 est accumulée en abondance. On sait maintenant que ces deux formes moléculaires sont codées par un gène unique (Nabeshima et al., 1984 ; Periasamy et al., 1984 ; Robert et al.,1984). Contrairement à ce que nous avons vu pour la $\beta$-tropomyosine, ici l'épissage alternatif implique la région $5^{\prime} \mathrm{du}$ gène. Chaque messager est transcrit à partir d'un promoteur spécifique associé au premier exon. Encore qu'aucune preuve expérimentale n'existe pour le moment, il est tentant d'imginer qu'il existe des facteurs spécifiques permettant la reconnaissance de ces promoteurs en fonction du stade de développement.

\section{Conclusions.}

Ce rapide survol des mécanismes moléculaires retrouvés au cours du processus de différenciation myogénique montre la diversité et la richesse des stratégies mises en œuvre pour permettre l'élaboration d'un phénotype différencié. 
II faut retenir que le passage de l'état précurseur à un état différencié implique le recrutement de toute une batterie de gènes appartenant au phénotype considéré. Ce recrutement peut aussi impliquer des gènes appartenant à un autre phénotype, en l'occurence le phénotype cardiaque. A ce sujet il est tentant de proposer que ceci est dû au fait qu'initialement les phénotypes squelettique et cardiaque appartenaient à un phénotype commun de type muscle strié. Egalement la cellule peut aussi utiliser un gène déjà transcrit mais en lui imposant un autre mode d'épissage de façon à produire un nouveau messager. Enfin, pour assurer les phénomènes de maturation, l'organisme peut faire appel à des séries de gènes liés dont l'expression subit une régulation coordonnée dans le temps ou utiliser des gènes uniques mais capables de subir des modes d'épissage différenciel dépendant du stade de développement.

$13^{e}$ Réunion du groupe Développement I.N.R.A., Cap d'Agde, 25-27 mai 1987.

\section{Références}

BANDMAN E., 1985. Myosin isoenzyme transitions in muscle development, maturation and disease. Int. Rev. Cytol., 97, 97-131.

BARTON P. J., ROBERT B., FISZMAN M. Y., LEADER D., BUCKINGHAM M. E., 1985. The same alkali myosin light chain gene is expressed in adult cardiac atria and in foetal skeletal muscle. J. Mus. Res. Cell Mot., 6, 461-475.

BERGSMA D. J., HAYWARD L., GRICHNIK J., SCHWARTZ R. J., 1986a. Regulation of actin gene expression during chicken myogenesis, 531-546. In C. EMERSON, D. FISCHMAN, B. NADAL-GINARD, M. A. Q. SIDDIOUI, Molecular biology of muscle development, vol. 29, Alan R. Liss Inc., New York.

BERGSMA D. J., GRICHNIK J. M., GOSSETT M. A., SCHWARTZ R. J., 1986b. Delimitation and characterization of cis-acting DNA sequences required for the regulated expression and transcriptional control of the chicken skeletal $\alpha$-actin gene. Mol. cell Biol., 6, 2462-2475.

BLAU H. M., CHIU C. P., WEBSTER C., 1983. Cytoplasmic activation of human nuclear genes in stable heterokaryons. Cell, 32, 1171-1180.

BLAU H. M., PAVLATH G. P., haRdeman E. C., ChIU C. P., SIlberstein L., WEBSTER S. G., MILLER S. C., WEBSTER C., 1985. Plasticity of the differentiated state. Science, 230, 758-766.

CERNY L. C., BANDMAN E., 1986. Contractile activity is required for the expression of neonatal myosin heavy chain in embryonic pectoralis muscle cultures. J. Ce/l Biol., 103, 2153-2161.

CHIU C. P., BLAU H. M., 1984. Reprogramming cell differenciation in the absence of DNA synthesis. Cell, 37, 879-887.

CROW M. T., STOCKDALE F. E., 1986. The developmental program of fast myosin heavy chain expression in avian skeletal muscles. Dev. Biol., 118, 333-342.

FISZMAN M. Y., KARDAMI E., LEMONNIER M., 1986. A single gene codes for gizzard and skeletal muscle $\alpha$-tropomyosin, 457-471. In C. EMERSON, D. FISCHMAN, B. NADAL-GINARD, M. A. Q. SIDDIQUI, Molecular biology of muscle development, vol. 29, Alan R. Liss Inc., New York.

GARNER I., MINTY A. J., ALONSO S., BARTON P. J., BUCKINGHAM M. E., 1986. A 5' duplication of the $\alpha$-cardiac actin gene in BALB/c mice is associated with abnormal levels of $\alpha$-cardiac and $\alpha$-skeletal actin mRNAs in adult cardiac tissue. EMBO J., 5. 2559-2567.

HARDEMAN E. C., CHIU C. P., MINTY A., BLAU H. M., 1986. The pattern of actin expression in human fibroblast $\times$ mouse muscle heterokaryons suggests that human muscle regulatory factors are produced. Cell, 47, 123-130. 
HELFMAN D. M., CHELEY S., KUISMANEN E., FINN L. A., YAMAWAKI-KATAOKA Y., $1986 . \quad$ Nonmuscle and muscle tropomyosin isoforms are expressed from a single gene by alternative RNA splicing and polyadenylation. Mol. cell Biol., 6, 3582-3595.

KONIECZNY S. F., EMERSON C. P., 1984. 5-Azacytidine induction of stable mesodermal stem cell lineages from $10 \mathrm{~T} 1 / 2$ cells : evidence for regulatory genes controlling determination. Cell, 38, $791-800$.

KONIECZNY S. F., EMERSON C. P., 1985. Differentiation, not determination, regulates muscle gene activation: transfection of troponin I genes into multipotential and muscle lineages of 10T1/2 cells. Mol. cell Biol., 5, 2423-2432.

LASSAR A. B., PATTERSON B. M., WEINTRAUB H., 1986. Transfection of a DNA locus that mediates the conversion of 10T1/2 fibroblasts to myoblasts. Cell, 57, 649-656.

LIBRI D., LEMONNIER M., MEINNEL T., FISZMAN M. Y., 1988. A single gene codes for the $\beta$-subunit of smooth and skeletal muscle tropomyosin in the chicken (à paraître).

MELLOUL D., ALONI B., CALVO J., YAFFE D., NUDEL U., 1984. Developmentally regulated expression of chimeric genes containing actin DNA sequences in transfected myogenic cells. $E M B O$ J., 3, 983-99.

MINTY A. J., ALONSO S., GUENET J. L., BUCKINGHAM M. E., 1982. A fetal skeletal muscle actin mRNA in the mouse and its identity with cardiac actin mRNA. Cell, 30, 185-192.

MINTY A., KEDES L., 1986. Upstream regions of the human cardiac actin gene that modulate its transcription in muscle cells : presence of an evolutionarily conserved repeated motif. $\mathrm{Mol}$. cell Biol., 6. 2125-2136.

MINTY A. J., HARDEMAN E., GUNNING P., BAINS W., BLAU H., KEDES L. H., 1986a. Regulation of actin gene expression in mammalian striated muscle, 507-519. In C. EMERSON, D. FISCHMAN, B. NADAL-GINARD, M. A. Q. SIDDIQUI, Molecular biology of musc/e development, vol. 29, Alan R. Liss Inc., New York.

MINTY A., BLAU H., KEDES L., 1986b. Two-level regulation of cardiac actin gene transcription : muscle-specific modulating factors can accumulate before gene activation. Mol. cell Biol., 6 , 2137-2148.

NABESHIMA Y. I., KURIYAMA Y. F., MURAMATSU M., OGATA K., 1984. Alternative transcription and two modes of splicing result in two myosin light chains from one gene. Nature (London), 308, 333-338.

NUdel U., GREenBerg D., ORDhAL C. P., SAXEL O., NEUMAN S., YAFFE D., 1985. Developmentally regulated expression of a chicken muscle-specific gene in stably transfected rat myogenic cells. Proc. nat. Acad. Sci. USA, 82, 3106-3109.

PERIASAMY M., STREHLER E. E., GARFINKEL L. I., GUBITS R. M., RUIZ-OPAZO N., NADALGINARD B., 1984. Fast skeletal muscle myosin light chains 1 and 3 are produced from a single gene by a combined process of differential RNA transcription and splicing. $J$. biol. Chem., 259, 13595-13604.

ROBERT B., DAUBAS P., AKIMENKO M. A., COHEN A., GUENET J. L., BUCKINGHAM M. E., 1984. A single locus in the mouse encodes both myosin light chains 1 and 3 , a second locus corresponds to a related pseudogene. Cell, 39, 129-140.

RUIZ-OPAZO N., NADAL-GINARD B., 1987. $\alpha$-tropomyosin gene organization Alternative splicing of duplicated isotype-specific exons accounts for the production of smooth and striated muscle isoforms. J. biol. Chem., 262, 4755-4765.

SCHWARTZ K., DE LA BASTIE D., BOUVERET P., OLIVIERO P., ALONSO S., BUCKINGHAM M., 1986. $\alpha$-skeletal muscle actin mRNA's accumulate in hypertrophied adult rat hearts. Circul. Res., 59, $551-555$.

SEILER-TUYNS A., ELRIDGE J. D., PATERSON B. M., 1984. Expression and regulation of chicken actin genes introduced into mouse myogenic and nonmyogenic cells. Proc. nat. Acad. Sci. USA, 81, 2980-2984.

SHANI M., 1985. Tissue-specific expression of rat myosin light chain 2 in transgenic mice. Nature (London), 314, 283-286.

SIDDIQUI M. A. Q., ZARRAGA A. M., NICHOLSON D., DESHPANDE A. K., SAIDAPET C., MENDOLA C., 1986. In C. EMERSON, D. FISCHMAN, B. NADAL-GINARD, M. A. O. SIDDIQUI, Molecular biology of muscle development, vol. 29, Alan R. Liss Inc., New York. 
SILBERSTEIN L., WEBSTER S. G., TRAVIS M., BLAU H. M., 1986. Developmental progression of myosin gene expression in cultured muscle cells. Cell, 46, 1075-1081.

TAYLOR S. M., JONES P. A., 1979. Multiple new phenotypes induced in 10T1/2 and 3T3 cells treated with 5-azacytidine. Cell, 17, 771-779.

TAYLOR S. M., JONES P. A., 1982. Changes in phenotypic expression in embryonic and adult cells treated with 5-azacytidine. J. cell Physiol., 111, 187-194.

WEYDERT A., DAUBAS P., LAZARIDIS I., BARTON P., GARNER I., LEADER D. P., BONHOMME F., CATALAN J., SIMON D., GUENET J. L., GROS F., BUCKINGHAM M. E., 1985. Genes for skeletal muscle myosin heavy chains are clustered and are not located on the same mouse chromosome as a cardiac myosin heavy chain. Proc. nat. Acad. Sci. USA, 82, 7183-7187.

WEYDERT A., BARTON P., HARRIS A. J., PINSET C., BUCKINGHAM M., 1987. Developmental pattern of mouse skeletal myosin heavy gene transcripts in vivo and in vitro. Cell, 49 , $121-129$.

WHALEN R. G., BUTLER-BROWNE G. S., GROS F., 1978. Identification of a novel form of myosin light chain present in embryonic muscle tissue and cultured muscle cells. J. mol. Biol., 126, 415-431.

YSRAELI J., ADELSTEIN R. S., MELLOUL D., NUDEL U., YAFFE D., CEDAR H., 1986. Musclespecific activation of a methylated chimeric actin gene. Cell, 46, 409-416. 